\title{
Protée
}

\section{Pratique et éthique}

\section{La théorie du lien}

\section{Jacques Fontanille}

Volume 36, numéro 2, automne 2008

Éthique et sémiotique du sujet

URI : https://id.erudit.org/iderudit/019016ar

DOI : https://doi.org/10.7202/019016ar

Aller au sommaire du numéro

\section{Éditeur(s)}

Département des arts et lettres - Université du Québec à Chicoutimi

ISSN

0300-3523 (imprimé)

1708-2307 (numérique)

Découvrir la revue

Citer cet article

Fontanille, J. (2008). Pratique et éthique : la théorie du lien. Protée, 36(2), 11-26. https://doi.org/10.7202/019016ar

\section{Résumé de l'article}

Le concept de « valeur " occupe une place centrale dans les sémiotiques d'inspiration linguistique, issues des travaux de Saussure, Hjelmslev, Benveniste et Greimas, et ce, au nom du principe différentiel qui les fonde. Parmi ces sémiotiques, la tendance massive des vingt dernières années, pour de multiples raisons, a consisté à traiter de ces valeurs dans une perspective esthétique. Sans renier les travaux de cette période féconde, qui a privilégié la voie phénoménologique, la relation au monde sensible, les esthésies et la culture artistique, de nombreux sémioticiens s'intéressent aujourd'hui à des discours, à des objets et à des pratiques qui relèvent de toute évidence d'un autre ordre de valeurs, qui impliquent des normes, une déontologie : en somme, des positions et des valeurs éthiques. L'approche sémiotique de la dimension éthique, dans la perspective ouverte par les recherches sur les pratiques et les interactions sociales, ne peut se cantonner à un inventaire des contenus sémantiques et modaux des valeurs. La forme de ces pratiques et de ces interactions sociales s'exprime essentiellement dans l'ordre syntagmatique, et le traitement sémiotique de l'éthique doit donc être syntagmatique et stratégique. La rhétorique générale de Perelman comporte à cet égard un ensemble de propositions très éclairantes et pourtant faiblement exploitées aujourd'hui : il s'agit, en l'occurrence, de la " théorie du lien ", des liaisons et des déliaisons, des ruptures et des freinages qui affectent les relations entre les éléments de la scène argumentative. Cet essai se propose de reprendre ces éléments, d'en formuler une articulation systématique et plus étendue que celle de Perelman, pour en rendre possible l'application aux pratiques sémiotiques en général. L'éthique des pratiques prend alors la forme globale d'une rhétorique des stratégies sociales.
Ce document est protégé par la loi sur le droit d'auteur. L'utilisation des services d'Érudit (y compris la reproduction) est assujettie à sa politique d'utilisation que vous pouvez consulter en ligne.

https://apropos.erudit.org/fr/usagers/politique-dutilisation/ 


\section{PRATIQUE ET ÉTHIQUE: LA THÉORIE DU LIEN}

JACQUES FONTANILLE

\section{INTRODUCTION: LA PLACE DE L'ÉTHIQUE EN SÉMIOTIQUE}

Les recherches sémiotiques, pour des raisons qui sont plus de sociologie des disciplines que proprement épistémologiques, se sont beaucoup occupées

d'esthétique et très peu d'éthique. La prééminence des objets «artistiques» dans les corpus préférés des sémioticiens et, plus généralement, le souci d'occuper une position académique dans le champ de la culture «légitime» les ont souvent conduits en effet à mettre en avant la réflexion sur l'émotion esthétique et sur les valeurs qui la suscitent.

Il existe pourtant plusieurs courants sémiologiques ou rhétoriques qui mettent en avant des préoccupations éthiques: on peut évoquer à ce titre, et parmi bien d'autres, la rhétorique générale de Chaïm Perelman, la socio-sémiotique d'Eric Landowski et, dans une certaine mesure, la sémiologie visuelle développée par Fernande Saint-Martin et ses collaborateurs, justement parce qu'elle résiste à une approche strictement esthétique de l'émotion artistique. Dans le même sens, les analyses télévisuelles de François Jost et plus particulièrement le modèle des «régimes de croyance» télévisuels impliquent des considérations plus éthiques qu'esthétiques.

Certes, les justifications épistémologiques du parti pris esthétique ne manquent pas, et la vogue des théories de la perception et de l'esthésie masque en fait trop souvent une prise de position implicite selon laquelle les valeurs sémiotiques seraient exclusivement articulées à partir de notre relation esthétique au monde sensible. Pour ce qui concerne la sémiotique post-greimassienne, le dernier livre écrit par Greimas seul, De l'imperfection, ou du moins les interprétations et les exploitations les plus fréquentes qui en ont été faites y sont sans doute pour quelque chose: les exemples qu'il utilise, littéraires et même souvent poétiques, et le style moins aride de ce livre ont encouragé des extrapolations esthétiques, et les commentateurs ont presque tous franchi le pas, par mégarde, entre l'esthésie et l'esthétique. Pourtant, les modalisations qu'il repère, à l'origine de la formation de l'existence sémiotique, les "devoir être» et les "pouvoir être», qui marquent la déhiscence entre le monde tel qu'il se présente et le sentiment d'existence, sont neutres à l'égard de la distinction entre éthique et esthétique. 
Cette position épistémologique, reposant en partie sur une approche phénoménologique très individualiste, néglige le plus souvent une dimension essentielle de la signification, la dimension sociale, et plus précisément celle des interactions pratiques; dans le champ académique anglo-saxon, le relatif déclin de l'ethnométhodologie confirme cette tendance. Les travaux les plus récents de Landowski montrent, $a$ contrario, que, dès qu'on se préoccupe des interactions sociales, l'éthique retrouve droit de cité dans les recherches sémiotiques; certes, Landowski, fidèle à la tradition greimassienne, fait une place importante à l'esthésie, mais il s'agit, en l'occurrence, de l'esthésie qui caractérise précisément le rapport à l'autre, et non la relation avec le monde sensible en général; on pourrait même avancer, dans cette perspective, que le cas général est l'esthésie du rapport à l'autre, et que la relation avec le monde sensible n'en est qu'un cas particulier: le «monde» est un «autre» parmi d'autres. Landowski apporte la preuve, à sa manière, que les valeurs sémiotiques prennent forme aussi dans le rapport à autrui, et que, dans ce cas, les esthésies gagnent une couleur éthique très marquée ${ }^{1}$.

On pourrait aussi avancer une autre hypothèse, de nature plus technique, qui expliquerait la prééminence tacite de l'esthétique sur l'éthique, et qui concerne cette fois la sélection des niveaux de pertinence sémiotique. En effet, l'analyse des effets esthétiques peut se concentrer sans dommage apparent sur les textes-énoncés, puisque, selon une tradition bien établie dans les herméneutiques occidentales, le «texte» a toujours le dernier mot en matière esthétique: le Beau y est objectivement fixé et inscrit, à travers des conditions textuelles de l'effet esthétique, et il suffit de savoir les reconnaître, c'est-àdire de disposer des instruments d'analyse appropriés pour en faire la description.

Or il en va tout autrement de l'éthique. Certes, on peut se donner une conception strictement textuelle de l'éthique, mais elle sera alors limitée aux contenus axiologiques, qu'ils soient saisis par une analyse isotopique de ces contenus ou à travers les opérations de la modalisation énonciative. Pourtant, cette réduction de l'éthique à l'axiologie et à la modalisation reste insatisfaisante, ne serait-ce que parce qu'on ne sait comment la distinguer de l'idéologie. Rappelons qu'à cet égard, l'idéologie était définie par Greimas comme l'articulation syntagmatique des valeurs et que ses «modèles" sont des "potentialités de procès sémiotiques ${ }^{2}$ : le repérage des isotopies axiologiques et des modalisations énonciatives, dans le texte même, ne saisirait, à ce titre, que l'idéologie du plan du contenu.

Un dernier doute vient enfin à l'esprit, et il tient à la place accordée à la rhétorique dans les réflexions sémiotiques. Passons sur le déni de scientificité et sur l'opprobre que les sciences du langage ont jeté en leur temps sur la tradition rhétorique. Plus intéressante est la sélection que Genette décrivait dans Figures III (1973) comme caractéristique de la «rhétorique restreinte». Car la rhétorique restreinte, même légèrement étendue, ne s'intéresse qu'aux tropes et aux figures, c'est-à-dire, au-delà des inventaires lassants et pédants dont elle est friande, aux valeurs esthétiques (Fontanille, 2003) inscrites dans le texte; or, ce qu'elle exclut, et qui a particulièrement été mis en évidence par Perelman tout au long de son œuvre, ce sont justement, et à la fois, les interactions argumentatives, la pratique persuasive et l'éthique de ces interactions et de cette pratique.

En somme, l'exclusion ou la réduction de l'éthique dans les recherches sémiotiques s'expliquerait aussi par le cantonnement de l'analyse immanente au texteénoncé: quand la sémiotique approche les phénomènes rhétoriques, elle sélectionne significativement parmi ces phénomènes ceux qui sont saisissables dans l'énoncé et ceux qui relèvent de l'esthétique. A contrario, dans la rhétorique générale, les phénomènes qui ne relèvent pas du texte ni de l'esthétique concernent très précisément la régulation des interactions sociales par le discours persuasif.

Pour pouvoir parler de l'éthique sans la réduire à l'idéologie et sans la confondre avec l'esthétique, il faut donc choisir un autre niveau de pertinence que celui du texte, et aborder la question sous l'angle des pratiques. Dans la hiérarchie des «plans 
d'immanence» et du parcours génératif de l'expression, comprenant figures-signes, textesénoncés, objets et pratiques, stratégies et formes de vie (Fontanille, 2007), il faut donc passer aux niveaux supérieurs à celui du texte-énoncé, au moins au niveau des pratiques et des stratégies.

\section{LA PRATIQUE PERSUASIVE ET SON ÉTHIQUE}

Commençons donc par examiner, à l'intérieur même de la pensée rhétorique, comment on passe, pour accéder à l'éthique, du texte à la pratique.

Dans la perspective du faire persuasif et de la production argumentative, la rhétorique propose une théorie générale qui comprend, outre les typologies d'arguments, de topoï, de figures et de tropes, des descriptions de procédures et de procédés, et de nombreuses règles pour la construction de l'ethos de l'orateur.

Or, la construction et le devenir de l'ethos ne peuvent se comprendre qu'à hauteur des pratiques, puisqu'ils outrepassent les limites du discours-énoncé. Si l'on s'en tient à l'acception courante telle que la définit l'étymologie, l'ethos recouvre l'ensemble des usages, des coutumes et des mœurs: l'ethos serait donc la forme régulière, reconnaissable et évaluable des pratiques.

Certes, même à hauteur des pratiques, le sens commun rechercherait volontiers l'éthique dans les contenus axiologiques qu'elles véhiculent, notamment dans les textes et les discours qu'elles manipulent ou produisent, mais laisserait du coup dans l'ombre leur «forme» propre, notamment ce qui fait qu'on peut y reconnaittre et y distinguer des "usages», des "mœurs", des «coutumes» ou des "traditions». Il nous faut donc définir plus précisément ce qui, dans la forme des pratiques, est plus spécifiquement d'ordre "éthique».

La rhétorique générale a proposé, sous la plume de Perelman $(1988)^{3}$, une des plus remarquables théories stratégiques qui soit, qui permet de définir et de spécifier l'ethos argumentatif et qui relève des procédures de la pratique persuasive ${ }^{4}$. C'est ce qu'on pourrait appeler la théorie $d u$ lien. Perelman, en effet, propose de rendre compte de l'ensemble des stratégies rhétoriques à partir de deux grands schèmes argumentatifs: la liaison et la dissociation. Ces deux schèmes s'appliquent à de nombreuses substances argumentatives au niveau du texte-énoncé, dont par exemple les notions (liaisons et dissociations, internes ou externes, dans ou entre les notions). Mais ils s'appliquent avec une puissance heuristique considérable à la praxis énonciative en acte, et notamment aux relations, dans les termes mêmes de Perelman, entre la personne, l'acte et le discours.

Et c'est cette même problématique qui permet de poser dans les termes les plus efficaces la question de l'éthique, puisque ce sont les liaisons et déliaisons ${ }^{5}$ entre l'acte, la personne et l'argument qui permettent de décrire les transformations de l'ethos, les aléas de la responsabilité et de l'imputation de responsabilité et les variations de la force d'engagement énonciatif. Les usages de la persuasion et les manières d'argumenter, selon Perelman, sont donc des figures et des séquences de la liaison et de la déliaison.

Quelles que soient les instances de la pratique qu'ils mettent en relation, ces liens entretiennent toujours un certain rapport aux valeurs, et ce rapport aux valeurs est notamment systématiquement actualisé lors des renforcements et affaiblissements des liens. En somme, la valeur de chaque tactique argumentative est exprimée et actualisée, et par conséquent principalement appréciable, à partir de ces modulations des liaisons et déliaisons. Les liens argumentatifs, selon Perelman, sont donc des liens porteurs d'axiologie.

On propose ainsi de définir l'éthique, dans un premier mouvement de généralisation, au-delà du cas particulier des pratiques argumentatives, du point de vue du fonctionnement syntagmatique des pratiques sémiotiques: ce sera l'ensemble des opérations portant sur ces «liens axiologiques».

L'éthique des pratiques s'apprécie donc non seulement, d'un point de vue sémiotique, à travers les contenus véhiculés et les croyances affichées en tant que tels, mais aussi à travers les procédures et les stratégies de liaison et de déliaison, et, globalement, à la forme reconnaissable des manipulations 
axiologiques qu'elles induisent. Les contenus axiologiques relèvent encore du niveau de pertinence du texte, alors que les opérations syntagmatiques impliquent la pratique toute entière. Nous pouvons à cet égard fixer les choses en distinguant deux approches complémentaires de l'éthique: (i) une approche textuelle, sémantique et paradigmatique, qui accède à l'éthique par une analyse isotopique et qui propose des «systèmes de valeurs" polarisés positivement et négativement; et (ii) une approche praxématique et syntagmatique, qui accède à l'éthique par une analyse des variations internes des processus pratiques.

Dans la perspective que nous avons choisie, nous rechercherons l'éthique sur la dimension syntagmatique des pratiques, tout spécialement dans les variations de l'ethos des pratiques. En faisant ce choix, nous réduisons volontairement mais provisoirement l'éthique à la «bonne forme syntagmatique» des pratiques, c'est-à-dire à une sorte d'éthologie des interactions sociales et des conduites pratiques.

Mais encore faut-il être en mesure de spécifier cette «forme syntagmatique». En effet, les liaisons et déliaisons de Perelman, en tant que procédures, affectent, on l'a déjà signalé, aussi bien les relations sémantiques entre notions ou entre parties de notions (donc dans le texte) que les relations entre acte, personne et argument (donc dans l'ensemble de la pratique, texte compris). La «forme syntagmatique» de la pratique doit donc être délimitée, et ses relations constitutives, circonscrites: la limite est celle de la scène prédicative élémentaire de la pratique et ses relations constitutives, celles qui assurent la consistance de cette scène (des actes, des actants et des propriétés des actes et des actants).

Notre propos est donc maintenant entièrement circonscrit: (i) il s'agit de tenter de généraliser les procédures du faire persuasif à l'ensemble des pratiques, (ii) en limitant l'examen aux schèmes de liaison et déliaison à l'intérieur de la scène prédicative des pratiques. Pour cela, nous devons (a) définir les instances de cette scène élémentaire de la pratique, (b) identifier la nature des «liens» entre les instances et (c) examiner le fonctionnement des opérations éthiques portant sur ces liens.

\section{LES INSTANCES DE LA SCĖNE INTERPRÉTATIVE}

La «scénarisation» de la pratique

La notion de «scène» est ici utilisée dans l'acception qui a cours en linguistique, notamment dans la syntaxe de Tesnière ou dans celle de Fillmore, celles mêmes à partir desquelles la linguistique moderne et la sémiotique narrative ont pu développer des théories actantielles. D'un point de vue déductif (et formel), la «scène» s'organise autour d'un prédicat et comprend le nombre d'actants (ou "places») nécessaires à l'actualisation de ce prédicat. D'un point de vue inductif (et perceptif), un événement ou un processus ont lieu, qui affectent un observateur; ils induisent, dans la perception de l'environnement, une discontinuité qui est aussi une demande de sens, et déclenchent chez le spectateur (qui peut être aussi l'acteur principal) une procédure de délimitation, la «scénarisation»: il agrège, autour de cette discontinuité, un certain nombre d'éléments nécessaires pour en construire le sens, opération au cours de laquelle il rencontre les limites de la pertinence, les «bords» de la scène.

Cette scène ne peut être ni phrastique, ni même textuelle; il s'agit de la «scène d'une pratique». Sans reprendre ici le débat sur l'immanence et le contexte, signalons seulement qu'une pratique est une «sémiotique-objet» qui peut comprendre (ou non) un ou plusieurs textes-énoncés, ainsi que d'autres éléments non textuels ${ }^{6}$. Dès que ces derniers sont intégrés à la scène d'une pratique (voir supra, la «scénarisation»), ils jouent alors des rôles d'actants et de circonstants du prédicat.

Traiter la pratique comme une sémiotique-objet, c'est s'engager à proposer un modèle qui permette d'articuler l'ensemble de ses constituants de manière homogène et cohérente. Concrètement, par exemple, quand la scène d'une pratique comprend un texteénoncé, ce dernier n'est pas supposé y occuper un statut à part, encore moins central, et le modèle recherché doit lui procurer une place de rang égal à 
celui des autres éléments pris en considération. En l'occurrence, la «scène prédicative» est ce modèle qui constitue le noyau définitionnel de la pratique, dans une définition intensive et non extensive dans la mesure où une pratique comprend d'autres éléments que ceux qui constituent la scène stricto sensu, notamment des éléments thématiques et figuratifs.

Les quatre instances de la scène persuasive

Concernant la composition de la scène prédicative, la position défendue par Perelman, au sujet de l'argumentation et du point de vue de la production des arguments et du faire persuasif, s'exprime essentiellement à propos de la construction de l'ethos de l'orateur, dont le ressort principal est le lien entre l'acte et la personne. Ce lien est discuté sur le fond d'une problématique traditionnelle en philosophie, celle de l'imputation de l'acte et de la responsabilité de la personne. Dans la perspective rhétorique, ce lien devient un enjeu stratégique et il fait l'objet de diverses manipulations que Perelman ramène globalement aux processus de renforcement et d'affaiblissement des liaisons et déliaisons.

Néanmoins, cette relation, dans la perspective même de Perelman, est incomplète, car, s'agissant des forces de liaisons et de déliaisons qui caractérisent les mouvements énonciatifs de la persuasion, il faut prendre en compte également l'énoncé produit (l'argument), ce qui permet d'examiner aussi les processus de liaison et de déliaison entre, d'une part, l'énoncé et l'acte et, d'autre part, l'énoncé et la personne. Perelman ne distingue pas formellement ces deux types de liens, mais le développement de son analyse les implique.

L'ethos de l'orateur est impliqué par le fait même que la proposition qu'il énonce est considérée non pas comme un énoncé universel, mais comme l'acte d'une personne, un acte imputable à un individu et qui puise une partie de sa valeur dans l'identité de cet individu.

Du côté de l'auditoire, l'évaluation de l'énoncé proposé implique donc au préalable un calcul d'«imputation» de l'acte argumentatif à la personne de l'orateur; inversement, du côté de l'orateur, la manipulation de la valeur des énoncés proposés est une manifestation de la «responsabilité» de la personne à l'égard de son acte. Mais, bien sûr, au cours du développement argumentatif, la valeur éthique induite par l'utilisation de tel argument rejaillira sur les suivants, et ainsi de suite, grâce à la médiation de l'ethos en construction dans la pratique toute entière.

Toujours dans la même perspective, il faudrait encore ajouter une quatrième instance, celle de l'auditeur ou énonciataire, qui non seulement est responsable de l'évaluation de l'ethos de l'énonciateur, mais qui, en outre, est un des éléments qui interagissent avec sa construction: en effet, selon que l'énonciataire est traité d'une manière ou d'une autre par l'énonciateur, soit indirectement, à travers le choix des arguments, soit directement, dans la représentation qu'en donne l'énoncé persuasif, l'ethos de l'énonciateur varie en conséquence, se renforce ou s'affaiblit, s'améliore ou se détériore.

Mais l'énonciataire ne joue un rôle dans la scène persuasive que dans la mesure où il est lui-même impliqué dans une autre pratique, sa propre sphère d'action ${ }^{7}$. En élargissant la perspective, on dira donc que, si la question de la valeur et de la force des arguments se pose, et si elle implique un autre actant, c'est parce qu'ils sont destinés à transformer une situation, et une croyance, à pousser cet actant à l'action, à infléchir sa décision ou sa position; il s'agit donc d'une axiologie performative, en ce sens que la valeur est dans ce cas actualisée dans les effets produits sur l'énonciataire et sur la scène à laquelle il appartient.

Il serait commode de définir cette autre scène praxique, englobant l'actant partenaire du premier, comme le "référent» de la pratique persuasive; mais une telle conception masquerait ce qui, justement, fait problème, à savoir que la modification de l'horizon référentiel est l'enjeu même de la pratique en cours, et que c'est en raison de cet enjeu que la valeur des énoncés persuasifs peut être appréciée.

De fait, cet horizon référentiel est bien la scène d'une «autre pratique», et la valeur des éléments de la scène persuasive est fonction de leur capacité à 
modifier cette autre scène. Au sens strict (Fontanille, 2007), cette connexion entre deux scènes est stratégique. La quatrième instance doit traduire ce pouvoir de connexion, entre les deux scènes, et de modification, de l'une par l'autre: c'est l'horizon stratégique.

Les instances praxiques

Qu'en est-il maintenant des instances de la pratique en général, au-delà des seules pratiques argumentatives?

Les deux premières instances propres au faire persuasif (l'acte et la personne, selon Perelman) sont immédiatement généralisables, puisqu'elles correspondent respectivement au "prédicat" central de la scène, et à l'un de ses actants, l'opérateur.

La troisième, l'argument énoncé, est propre aux pratiques dites "discursives", qui comprennent au moins la production d'un texte-énoncé. Ce dernier y joue précisément le rôle de l'actant objet, un objet produit par l'acte et l'opérateur. Ces considérations nous renvoient à la conception classique de la transformation, dont on sait bien que l'actant-objet, au niveau d'abstraction qui est le sien, ne correspond à des «figures-objets» que dans un nombre limité de cas. L'acte praxique ne consiste pas nécessairement en un acte de production d'objets, ni même de conquête d'objets. Il existe des pratiques qui ne visent qu'à la modification d'une situation, ou qui ne visent même qu'à résister à une modification en cours.

Mieux vaudrait, en l'occurrence, comme l'a fait la grammaire des cas en son temps, parler d' "objectif" plutôt que d'objet.

La quatrième instance identifiée ci-dessus, l'«autre scène» qui comprend l'actant partenaire, doit être identifiée à l'ensemble des autres pratiques avec lesquelles la pratique en cours entre en interaction: ce sont justement ces interactions qui caractérisent la dimension stratégique (Fontanille, 2006) et on peut alors invoquer, pour les pratiques en général, un actant qui serait l'«horizon stratégique».

La composition de la scène praxique peut donc être arrêtée à quatre instances, trois instances actantielles et une instance prédicative:
- l'acte;

- l'opérateur;

- l'objectif (qui peut être un «résultat»);

- l'horizon stratégique, et notamment l'«autre scène", sachant que cette dernière comprendra également un acte et des actants.

Comme toute composition canonique, celle-ci est soumise à des variations et à des syncrétismes, en particulier le syncrétisme entre l'objectif et l'horizon stratégique, mais comme les cas ne manqueront pas où l'objectif propre à la pratique en cours ne se confond pas avec la gestion des interactions stratégiques, la distinction doit être maintenue. En outre, dans la mesure où l'analyse de la dimension éthique va justement porter sur les relations entre les instances et sur les liaisons et déliaisons qui les affectent, le syncrétisme entre instances est précisément l'un des cas de figure qui intéressent l'analyse éthique, comme phase ultime du renforcement du lien, jusqu'à l'inclusion d'une instance dans l'autre.

Tout comme dans le faire persuasif, la valeur des productions pratiques dépend de l'ethos de l'opérateur, mais plus généralement de l'ethos de la scène praxique, qui se construit dans les relations entre l'opérateur, l'acte, l'objectif et l'autre scène. L'effet axiologique du lien entre l'acte et les trois autres instances est donc réciproque, interactif et cumulatif: la valeur de la production pratique modifie l'ethos de l'opérateur (il est augmenté ou diminué selon que la production est acceptable ou pas, honorable ou pas, etc.) et l'ethos de l'opérateur modifie la valeur de sa production, à proportion de sa propre crédibilité, de son autorité, de son engagement, etc.

Le noyau explicatif de cette contagion axiologique est la structure même de la scène prédicative, le prédicat étant au centre, et les autres instances, définies par la nature de celui-ci; en d'autres termes, c'est en raison de la force de liaison de l'acte même (cette force de liaison que Tesnière appelait la «valence», comme pour les électrons autour du noyau atomique) qu'une telle diffusion axiologique peut se 
produire entre les différentes instances. Nous y reviendrons bientôt, mais, d'ores et déjà, nous sommes en mesure d'affirmer que l'ethos qui nous intéresse est non seulement l'ethos de l'opérateur pris isolément, comme chez Perelman, mais aussi l'ethos de la scène pratique dans son ensemble, c'est-à-dire l'ethos qui se dessine dans la totalité des liens constitutifs de la scène.

La nature des liens entre instances

1) L'acte et sa "valence»

L'acte d'effectuation ne peut se produire qu'en présence des trois instances impliquées: l'actant opérateur, l'objectif et l'autre scène. L'acte implique ses actants, comme conditions de possibilité et de réalisation. C'est le résultat de la scénarisation globale de la pratique.

D'un côté, le lien avec l'opérateur est construit par des calculs d'imputation de l'acte à un actant et les degrés de croyance de cette imputation. Les régimes de croyance associés à ces calculs d'imputation peuvent construire des liens "causaux", "fictionnels", «magiques», etc., selon que les effets sur l'autre scène seront considérés comme directs, indirects, indiciels, symboliques, etc.

De l'autre côté, le lien entre l'acte et l'objectif peut être évalué selon les degrés de la modalisation existentielle: l'objectif, en effet, peut être saisi dans la perspective finale de la réalisation, mais aussi dans toutes les phases intermédiaires, comme la virtualisation, la potentialisation ou l'actualisation.

Enfin, d'autres variations sont envisageables, notamment des ellipses ou des syncrétismes d'instances. Comme nous l'a enseigné Tesnière, en effet, les valences prédicatives peuvent être saturées ou non, et les instances peuvent fusionner ou être virtualisées. Nous y reviendrons.

\section{2) L'objectif et le résultat}

L'objectif, a fortiori quand il est réalisé (voir le résultat), peut comporter des traces de l'acte et des manifestations de l'actant opérateur. À ce titre, il est l'«expression» de l'un et de l'autre.
Lobjectif et le résultat expriment l'acte chacun à leur manière; en effet, cette distinction entre les deux modes d'existence de l'actant-objet implique tout particulièrement la confrontation entre les effets "projetés» et les effets «induits», et il s'agit donc, entre autres, d'une problématique principalement volitive. Mais le lien entre les "effets projetés" et les "effets induits» est assuré notamment par un «croire faire», nécessaire à la réalisation de l'acte.

Les «effets projetés» reposent sur les objectifs et les "effets induits", sur les résultats; les deux, objectifs et résultats, sont la conséquence d'une analyse syntagmatique de l'actant-objet. Leur différence de statut modal (l'un est virtuel ou potentiel, l'autre est actuel ou réel) implique ipso facto une différence de contenu, en même temps qu'une solidarité inaliénable; le résultat ne peut être apprécié qu'en fonction de l'objectif, et, néanmoins, l'écart qui les sépare est irréductible; l'objectif, en se réalisant, se déplace, s'appauvrit ou se complexifie; inversement, le résultat ne réfléchit jamais complètement l'objectif. Il y a donc, à l'intérieur même de cet actant-objet, un lien interne qui est lui-même modulable; pour simplifier la présentation générale du modèle, on considérera néanmoins qu'il s'agit d'une seule instance, comprenant des variantes modales.

Par ailleurs, l'objectif et le résultat expriment non pas directement l'opérateur, mais son ethos, en tant qu'évaluation de sa compétence modale et thématique; ce lien d'expression induit l'ensemble des problématiques de la véridiction et des stratégies afférentes: une autre croyance est en jeu, un «croire être» qui se manifesterait du fait même de l'actualisation du faire.

\section{3) L'opérateur et son ethos propre}

Dans la scène prédicative, l'opérateur est caractérisé par une compétence modale, car il est investi par les propriétés modales de l'acte, notamment par le statut existentiel de ce dernier (virtuel, actuel, potentiel ou réalisé), qui se traduit par une modalisation factitive du lien entre acte et opérateur (vouloir, devoir, savoir, pouvoir). Il est 
également défini par une compétence thématique, dans la mesure où la thématique de l'objectif (ou du résultat, selon qu'elle diffère ou non de celle de l'objectif) constitue elle aussi un investissement sémantique du sujet. Ce dernier reçoit donc une double détermination sémantique: une détermination thématique qui provient de l'objectif et une détermination modale qui provient de l'acte.

Cette dimension de l'éthique est la seule dont on trouve trace dans la tradition sémiotique d'inspiration greimassienne, en particulier sous la plume de Daniel Patte: il proposait en effet, dans Sémiotique II (1986), de compléter l'inventaire des modalités par celui des modalités éthiques, en les fondant sur les modalités déontiques (devoir faire et pouvoir faire, selon lui), associées au croire. Deux systèmes modaux en découlent, celui du "croire devoir faire» et celui du "croire pouvoir faire»; la déclinaison catégorielle du premier permet de définir l'«engagement», le «détachement», l'«intérêt» et l'«indifférence», alors que celle du second articule quatre types de «sentiments»: les sentiments de «compétence» et d'«incompétence», d'«aptitude» et d'«inaptitude». Cette proposition n'a pas été reprise, en raison de son caractère mécanique et faiblement heuristique ${ }^{8}$, mais elle signale à juste titre un des lieux d'analyse syntagmatique de la dimension éthique des discours.

Globalement, le rapport de l'opérateur à son propre ethos, sous ses deux dimensions, modale et thématique, est régi par un troisième type de croyance, celui même que Daniel Patte proposait de réserver au devoir faire et au pouvoir faire: il s'agit de la croyance qui fonde l'assomption éthique (le minimum requis pour pouvoir parler de la «responsabilité» de l'opérateur); cette croyance portant sur l'identité thématico-modale est plus ou moins forte ou faible, et les variations d'assomption qui en découlent affectent plus particulièrement les liens internes qui associent les éléments de l'ethos entre eux.

Dès lors, l'identité thématico-modale de l'actant peut être soumise à plusieurs types de variations, en trois directions: (i) les unes touchent à la cohésion de l'identité (entre parties, entre étapes); (ii) d'autres touchent à sa congruence (entre la thématique et les modalités); (iii) d'autres enfin touchent à sa cohérence (dans un parcours praxique global, en fonction, notamment, de la mémoire des pratiques antérieures). Les formes de ces variations sont en partie prévisibles, d'un point de vue syntagmatique, puisque, dans un parcours praxique ou stratégique, la cohésion, la congruence et la cohérence de l'identité peuvent obéir à deux régimes ${ }^{9}$ : le régime «idem», pour une identité affirmée par répétition, et le régime «ipse», pour une identité affirmée par persévérance.

\section{4) L'autre scène et l'horizon stratégique}

L'autre scène est celle de la pratique (ou des pratiques) que la pratique en cours s'efforce de modifier, ou avec lesquelles elle entre en interaction (la forme minimale canonique de cette interaction peut être une relation entre programme d'usage et programme de base). Le discours politique, par exemple, vise à infléchir les pratiques électorales; les pratiques liturgiques s'efforcent de présentifier une scène originaire, en général celle d'un sacrifice; l'interprétation musicale fait de même avec la composition musicale; la dégustation d'un vin a pour horizon un ensemble de pratiques commerciales, gastronomiques ou classificatoires.

La nature des liens entre les instances de la scène en cours et celles de l'autre scène est difficilement prévisible, et on peut seulement en indiquer quelques cas, à titre d'illustrations. Par exemple, l'opérateur peut se placer dans un rapport d'identification avec tel ou tel actant de l'autre scène; ou encore, l'objectif de la pratique considérée peut être d'extraire et de présenter des règles de fonctionnement de l'autre scène, comme c'est le cas de toute pratique scientifique; les pratiques thérapeutiques s'efforcent elles aussi de dégager, explicitement ou implicitement, des «lois» et des «normes» qui caractérisent les pratiques du corps au quotidien; on a alors affaire à une relation de type méta-sémiotique.

Globalement, la relation entre les deux scènes oscille entre, d'une part, la manipulation, qui 
programme les modalisations factitives de l'actant de l'autre scène et installe les rôles passionnels et thématiques adéquats, et, d'autre part, l'ajustement, qui agit plus spécifiquement sur les relations spatiotemporelles, sur les harmonies aspectuelles et rythmiques entre les deux scènes ${ }^{10}$.

En outre, on peut raisonnablement supposer que la relation entre les deux scènes est régie dans la plupart des cas par leur appartenance commune à une séquence temporelle et narrative, voire à une séquence canonique, où la pratique en cours correspond à l'une des grandes étapes du schéma narratif; mais ces positions canoniques ne constituent qu'un des multiples cas de liaison entre les deux scènes.

Le modèle triangulaire qui se dessine permet donc de rendre compte de l'articulation, entre les trois instances actantielles et l'acte, et de la nature des «liens» que les stratégies rhétoriques auront à renforcer ou à affaiblir:

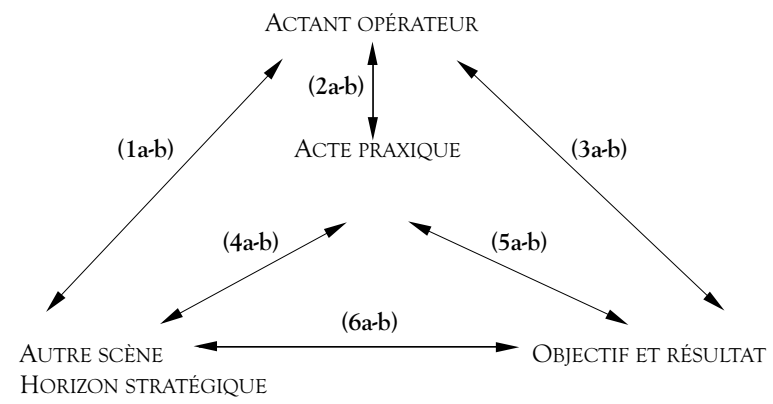

Nous obtenons ainsi six liens de base, comprenant chacun deux directions, et sur lesquelles portent deux opérations (liaisons et déliaisons), ce qui fait en tout vingt-quatre possibilités.

\section{LA TYPOLOGIE DES LIENS ET DE LEURS VARIÉTÉS}

Les six «liens» réciproques de base (les douze directions) doivent maintenant être définis plus précisément:

(1a) L'opérateur vise l'autre scène, celle qu'il doit en fin de compte contribuer à transformer, et il doit pour cela s'en donner une représentation transformable: il en identifie les variables et les tensions instables, sur lesquelles faire porter les modifications.

(1b) L'autre scène offre des possibilités de positionnement stratégique (identification, schématisation méta-sémiotique, hiérarchisation et enchâssement syntagmatiques entre pratiques, etc.) à l'opérateur.

(2a) La présence de l'opérateur, en tant qu'actant, est requise pour la réalisation de l'acte. Cette condition étant modalisable, et son effet, évaluable, l'opérateur peut de ce fait assumer ou ne pas assumer ses actes interprétatifs et il manifeste ainsi différents degrés de responsabilité.

(2b) L'acte est imputable à l'opérateur, et cette imputation est elle-même soumise à une modalisation existentielle du prédicat, qui peut être saisi, du point de vue de l'opérateur luimême, comme virtuel, actuel, potentiel ou réel, et, en fonction de cette modalisation existentielle, l'opérateur, à travers la relation d'imputation, sera lui-même identifié par son vouloir, son devoir, son pouvoir, son savoir ou son croire.

(3a) L'opérateur exprime son ethos à travers l'objectif qu'il vise et le résultat qu'il atteint.

(3b) Inversement, l'objectif et son résultat thématisent l'opérateur.

(4a) L'acte transforme l'autre scène, en modifie les conditions initiales et les équilibres internes; il procède également par ajustements spatiotemporels, rythmiques et aspectuels entre la scène à laquelle il appartient et cette autre scène.

(4b) L'autre scène et l'horizon stratégique dans son ensemble offrent à l'application de l'acte praxique une diversité de composants et de "zones d'impact", sélectionnés notamment par focalisation.

(5a) L'acte praxique vise un objectif et produit un résultat, mais, en retour, objectif et résultat en sont la condition et le critère de réalisation (en tant qu'actant-objet).

(5b) Inversement, objectif et résultat manifestent l'acte, non seulement comme aboutissement (d'un point de vue aspectuel), mais aussi comme 
lieu d'inscription de ses traces. Le lien modulable est donc ici la rémanence de l'acte dans son résultat.

(6a) L'autre scène et l'horizon stratégique à transformer sont articulés avec l'objectif et le résultat, dans l'exacte mesure où cette articulation permet d'apprécier l'efficience de la pratique: ces articulations, directes ou indirectes, de même polarité axiologique ou de polarité différente, de renforcement ou d'affaiblissement, caractérisent en effet la capacité du résultat produit par un acte à infléchir significativement (et dans quel sens?) l'horizon des autres pratiques associées; le lien modulable est donc celui de l'efficience.

(6b) L'objectif adopte à l'égard de l'autre scène des positions stratégiques diverses (méta-sémiotiques, connotatives, fictionnelles, hiérarchiques) visant à renforcer ou affaiblir le lien d'efficience; comme ces positions stratégiques visent globalement à sélectionner ou à établir la possibilité même d'un ajustement entre les deux scènes, on peut considérer que le lien modulable est dans ce cas un lien de congruence.

Les «liens» qui tissent la structure solidaire de la scène praxique sont donc non pas des propriétés ad hoc, mais des relations sémiotiques plus générales, qui constituent en somme le réseau conceptuel de la sphère éthique: représentation, positionnement stratégique, responsabilité, imputation, modalisation, expression, thématisation, ajustement, sélection, rémanence, efficience, congruence en sont les principaux.

Il est à noter par ailleurs que les théories de la communication ${ }^{11}$ font état, traditionnellement, de deux autres types de liens, le "contact» et le «canal». La communication est un cas particulier des pratiques sémiotiques et, par conséquent, ces deux "fonctions" ne sont pas directement transposables dans la perspective ici adoptée. Le canal n'a pas sa place dans la scène praxique, dans la mesure où, simple support et moyen matériel de l'échange, il correspond, dans l'analyse des pratiques, au substrat sur lequel elles opèrent, notamment quand il s'agit d'opérations qui visent à transformer un segment du monde naturel et ce substrat appartient aux déterminants figuratifs et sensibles de la pratique, et non à la scène praxique stricto sensu.

Il en va tout autrement du «contact», à partir duquel est définie la «fonction phatique». Le phatique, en effet, est un type de lien spécifique qui se noue entre les partenaires de l'échange et, à ce titre, il peut être considéré comme un cas particulier du lien qui s'établit, dans notre modèle, entre l'opérateur et son horizon stratégique, sachant que, par ailleurs, cet horizon comprend les partenaires de la pratique en cours. Le lien phatique, de nature affective et somatique, est donc un cas d'espèce des relations que l'opérateur entretient avec les autres acteurs engagés dans la même pratique.

\section{MODULATIONS DU LIEN: RUPTURES ET FREINAGES}

Les forces de liaison dans la scène praxique

Pour ce qui concerne les principes transposables, les procédures de Perelman (les «schèmes argumentatifs") sont composées d'opérations classées en deux types: d'une part, la "rupture», qui inverse le mode de raisonnement et fait passer de la liaison à la déliaison, et réciproquement; d'autre part, le "freinage", qui affaiblit la liaison ou la déliaison. La «rupture» opère sur les tensions éthiques en les inversant et en les déplaçant; quant au "freinage», il oppose à la tension principale une tension contraire, il introduit une distance dans un lien, ou un rapprochement sur un fond de déliaison.

Par conséquent, toute la valeur opératoire du modèle sémiotique de l'éthique reposera sur les opérations de freinage et de rupture. Notre objectif, en effet, est d'identifier les phénomènes d'ordre syntagmatique et énonciatif (et non d'ordre sémantico-idéologique) qui produisent des valeurs éthiques. Par ailleurs, nous sommes partis du principe que ces valeurs ne pouvaient être saisies que dans les «mouvements» qui les transforment, dans les modifications des liens, puisque c'est par ces mouvements mêmes que les valeurs éthiques s'actualisent pour les partenaires des interactions. Par 
exemple, c'est au moment même où un opérateur s'efforce d'adopter une attitude plus détachée à l'égard de son objectif que ses partenaires peuvent repérer à la fois que quelque chose le relie à cet objectif et qu'il est en train d'en modifier la valeur, voire de falsifier son rapport axiologique à cet objectif. Dans ce cas, c'est un lien d'assomption qui est affaibli.

L'ensemble des douze liens définis ci-dessus, que ce soit dans le sens de la liaison ou dans celui de la déliaison, peuvent chacun faire l'objet de renforcements et d'affaiblissements ou, pour reprendre la terminologie de Perelman, de "ruptures» et de «freinages», ce qui porte les vingt-quatre possibilités envisagées à une infinité de cas de figure et de degrés. Il est question ici non pas de les examiner un par un, mais de préciser leur statut et d'en donner quelques exemples.

On pourrait être tenté de reconnaître, dans les opérations élémentaires, les deux schèmes de base, l'équivalent de la conjonction (liaison) et de la disjonction (déliaison), transposées à hauteur des pratiques; allons plus loin: il y a même quelque chose, dans leur définition et dans leurs modes de fonctionnement, qui les apparenterait à l'embrayage et au débrayage, si l'embrayage et le débrayage pouvaient s'appliquer à tous types de faire, et pas seulement au faire énonciatif ${ }^{12}$. Pourtant, ces ressemblances sont trompeuses, et la réduction qui pourrait en découler, particulièrement fâcheuse. Et il y a à cela plusieurs raisons.

Pour commencer, la liaison et la déliaison opèrent, de manière graduelle, sur des tensions dont elles modifient la force, sur des équilibres qu'elles perturbent ou renforcent, autorisant en cela l'inversion ou la modération des tensions en cours. De ce point de vue, elles se rapprocheraient plus du modèle socio-sémiotique de l'«union» et de la "contagion" selon Landowski (2004), que de celui de la «jonction» selon Greimas (1970).

En outre, contrairement au débrayage et à l'embrayage, et même quand elles affectent des pratiques verbales et énonciatives, la liaison et la déliaison n'opèrent pas entre des plans d'énonciation différents, encore moins entre des scènes d'énonciation différentes, comprenant des actants distincts. Le brayage gère principalement la hiérarchie entre plusieurs scènes d'énonciation, ainsi que les «entrées» et les «sorties» de la scène d'énonciation principale, comme chez Benveniste (1966), entre l'énonciation du discours et celle de l'histoire.

A fortiori, s'agissant de pratiques non verbales, non seulement la liaison et la déliaison ne concernent pas les rapports entre plans d'énonciation, puisqu'il n'y a pas "énonciation", mais en outre elles opèrent évidemment à l'intérieur d'une même scène praxique, entre les éléments constitutifs de cette scène, voire, dans certains cas, «à l'intérieur» d'un seul et même actant. Ce n'est que dans le cas de l'horizon stratégique que pourrait être envisagée une relation entre deux scènes distinctes, mais nous avons montré que même cet «horizon» appartient à la scène principale. Les liaisons et déliaisons, par conséquent, telles que nous venons de les circonscrire, ne sont pas en mesure de rendre compte de variations de points de vue, ou de conflits de positions idéologiques, car elles ne sont conçues que pour rendre compte de la dimension éthique.

Il nous faut donc poser l'existence autonome d'un type d'opérations propres à la scène prédicative des pratiques, qui touchent à la force du lien institué par la valence (entre prédicat et actants) et par la sémiose même (entre expression et contenu). La «force du lien» syntagmatique ne se manifeste que s'il est soumis à des fluctuations; on sait par exemple, en grammaire phrastique, que certaines opérations de déplacement ou d'extraction sont plus ou moins acceptables, selon qu'elles affectent des liens phrastiques «forts» (dans le noyau prédicatif) ou «faibles» (dans l'entour circonstanciel de ce noyau), et c'est seulement au moment de la tentative d'extraction qu'on peut vérifier la force de liaison qui assure la solidarité entre les éléments de la scène prédicative.

La force de liaison propre à la valence est la seule explication possible, par exemple, des variations métonymiques, qui permettent d'évoquer l'une des instances d'une scène prédicative par l'intermédiaire 
d'une autre instance: la tension globale entre les valences prédicatives semble se relâcher en production et se rétablir au moment de l'interprétation de la métonymie. Mais cette force ne s'exprime pas seulement dans le discours: il n'y a pas en effet que dans les textes que l'ouvrier s'identifie à son outil, par métonymie; dans la pratique «in vivo" même, il peut s'identifier à son outil, ou à son métier, ou à son atelier; et même si les implications sont différentes, le principe structurel sémiotique qui sous-tend de tels phénomènes est le même, et ils révèlent tous, et de la même manière, la force de liaison propre à toute scène praxique. La force de liaison propre à la sémiose est aussi soumise aux mêmes variations tensives, qui en révèlent la pertinence: dans le théâtre de Ionesco, par exemple, la prolifération répétitive des figures du plan de l'expression, qu'il soit verbal ou objectal, dans le texte ou dans les indications de mise en scène, "vide» le plan du contenu de toute substance sémantique.

La consistance de la scène praxique

La «force de liaison» est donc un concept hypothétique, dont on ne peut tester la valeur opératoire qu'en cherchant à la modifier. Il faut par conséquent lui attribuer des conditions d'actualisation qui, à ce qu'il semble, pourraient être au nombre de deux: (i) elle ne se manifeste qu'en raison de la solidarité nécessaire entre les éléments constitutifs d'une scène: en ce sens, elle n'est qu'un autre nom pour la stabilisation iconique ${ }^{13}$ de la scène prédicative (le produit de la scénarisation, voir supra); (ii) toute modification d'un lien entraine par compensation la modification d'un ou de plusieurs autres liens et, si ce n'est pas le cas, la stabilité iconique de la scène est compromise, ainsi que le succès éventuel de la pratique en cours; il y aurait donc à cet égard une «homéostasie» des liens.

Si la jonction intéresse les relations entre états, voire entre situations narratives, si le brayage concerne les relations entre plans et scènes d'énonciation, alors il faut admettre que les opérations de liaison et de déliaison touchent à une autre dimension des structures sémiotiques: leur «consistance».
La consistance est la force homéomère qui solidarise les parties des structures anhoméomères. L'ensemble des éléments constitutifs des scènes prédicatives sont en effet des parties formant un tout, grâce aux «liens" que nous avons examinés plus haut, mais nous faisons maintenant l'hypothèse d'une solidarité entre tous ces liens, la consistance qui assure la reconnaissance et le fonctionnement global de la scène praxique.

Les «ruptures» et les «freinages» éprouvent la consistance de cette totalité, en affectant chacun des liens qui la constituent. La «rupture» transforme une liaison en déliaison (la déliaison est encore un lien, même s'il est négatif) et le freinage affaiblit l'un ou l'autre - à quoi il faudrait ajouter le "renforcement» des liens existants. Ces opérations ne remettent pas en cause l'existence même des liens ni de la scène en tant que telle; elles jouent de l'«élasticité» de ces liens, pour y faire apparaître des variations éthiques ${ }^{14}$.

En somme, l'ethos de la scène pratique est un autre nom pour la plasticité de sa forme et de sa stabilité iconique. Et l'«éthologie» que nous visons est une étude de ces variations plastiques, à l'intérieur d'une forme iconique éprouvée mais maintenue.

\section{QUELQUES EXEMPLES}

Les figures du "prestige» ou de l'«autorité» contribuent, c'est un exemple classique, au renforcement du lien entre l'acte et l'actant, puisque c'est la compétence ou, plus généralement, l'ethos affichés par l'opérateur qui légitiment l'acte. Il faudrait à cet égard distinguer deux formes de la confiance: l'une reposant sur des chaînes de garanties «institutionnelles» et l'autre reposant sur une connivence sociale (à la limite, inter-individuelle) intéressée. Dans le premier cas, le renforcement du lien entre l'acte et la personne est suscité par le nombre et la hiérarchie des garants, hiérarchie réglée par ailleurs dans une institution sociale; dans le second cas, c'est le lien entre l'opérateur et l'«autre scène", lien fondé sur l'intérêt, qui est renforcé par la charge passionnelle. Bien sûr, dans chacun des cas, l'un des deux liens est renforcé au détriment de l'autre. 
Dans ce dernier cas, la collusion entre l'opérateur et l'autre scène, dont le lien est renforcé notamment par l'intérêt, pourra être évaluée négativement, et elle sera alors considérée d'un point de vue éthique comme une "compromission".

On peut se rapporter, en l'occurrence, aux analyses de Perelman consacrées à la "prévention": dans les limites de la pratique argumentative, la prévention affaiblit la force des arguments, dans la mesure où la conclusion visée par l'orateur semble pour lui déjà acquise et ne peut être considérée comme résultant de la bonne forme de l'argumentation, encore moins d'une négociation avec l'auditoire. La "vérité» obtenue étant non pas construite dans l'interaction, mais posée implicitement avant même cette interaction, sa valeur en est d'autant affaiblie; la prévention, en tant que figure argumentative, semble alors manifester, pour l'auditoire, un intérêt personnel trop marqué de l'orateur pour la conclusion qu'il vise: au-delà de la prévention, c'est donc bien la force excessive du lien entre l'orateur et l'«autre scène» qui est en cause.

La compromission agit de la même manière: l'intérêt de l'opérateur, engagé dans la modification ultime de l'autre scène et de l'ensemble de l'horizon stratégique, affaiblit la valeur de sa pratique, ainsi que sa crédibilité dans la réalisation de l'acte principal. En somme, la «morale» sous-jacente, en l'occurrence, voudrait que l'opérateur soit à la fois engagé dans son objectif, mais qu'il ne soit pas le bénéficiaire direct des conséquences de la transformation de l'horizon stratégique. D'autres «morales" préconiseraient le contraire, c'est-à-dire un lien de collusion renforcé avec l'horizon stratégique et un lien affaibli avec l'acte et l'objectif. Dans tous les cas, les variations de l'ethos opèrent "à somme nulle», c'est-à-dire que chaque renforcement de l'un des liens se fait au détriment d'un ou de plusieurs autres.

Un des cas typiques de la déliaison ou du freinage de la liaison a déjà été évoqué: c'est celui qui consiste à considérer l'objectif comme "détaché» de son producteur. Bien des débats sur les rapports entre l'homme et l'œuvre, bien des distinctions entre «effets voulus» et "effets induits» reposent sur ce type de freinage, ou postulent même une indépendance radicale entre l'objectif de l'opérateur, et a fortiori son résultat et ses prétendues «intentions». Les jugements de maladresse ou d'inconséquence participent de cette problématique.

S'agissant du lien d'imputation et de responsabilité entre l'opérateur et l'acte, les freinages et les ruptures de la liaison convoquent eux aussi toute une gamme de jugements positifs ou négatifs. La tradition classique française, en valorisant la figure de l'«honnête homme», visait très exactement ce lien éthique d'imputation: l'honnête homme, en effet, est réputé «ne se piquer de rien», c'est-à-dire n'entretenir aucun engagement passionnel particulier avec les actes qu'il accomplit. Il en va tout différemment de l'«homme sans qualité» de Musil ou même du «Swann avant Odette» de Proust, qui n'accordent aucune valeur aux objets qu'ils manipulent et consomment, alors qu'ils sont susceptibles de s'investir intensément dans tous les menus actes qui composent leurs pratiques quotidiennes. Ils entretiennent, en somme, à la fois une relation très affaiblie avec l'objectif et une relation forte avec l'acte. En revanche, l'homme qui ne se pique de rien affecte de n'avoir qu'un lien relâché avec ses actes, tout en assumant ses objectifs.

Mais il existe une autre version du même type de freinage, qui confine à la rupture: c'est celle de l'acte manqué, l'acte qui semble complètement échapper à son auteur. De fait, toute la tradition interprétative de l'acte manqué et du lapsus, notamment celle, analytique, initiée par Freud dans Psychopathologie de la vie quotidienne, vise pourtant à reconstituer un lien d'imputation entre l'acte et l'opérateur. Mais il s'agit alors d'une imputation sans responsabilité: rappelons que, dans notre typologie, le lien 2a (opérateur $\rightarrow$ acte) est celui de la responsabilité et le lien $2 \mathrm{~b}$ (acte $\rightarrow$ opérateur) est celui de l'imputation; dans le modèle canonique de la scène praxique, ils sont réputés inverses et solidaires l'un de l'autre; dans le modèle analytique de l'acte manqué, le premier est affaibli, voire rompu, et le second est renforcé. On pourrait 
même considérer que l'ensemble du raisonnement tenu par Freud, notamment à propos des «obstacles» rencontrés par les intentions de l'auteur de l'acte, exploite lui aussi les figures éthiques du freinage et de la rupture.

Cette analyse nourrit par ailleurs bien des débats judiciaires, puisque l'établissement de ces deux liens inverses est une condition préalable de tout jugement, et que même deux types d' "experts" différents en sont chargés: d'un côté, le lien d'imputation est en charge des enquêteurs (police, etc.) et, de l'autre, le lien de responsabilité est en charge des psychologues; c'est ensuite au juge qu'il revient d'apprécier l'équilibre entre les deux liens et de prononcer, en préalable à toute décision, un jugement éthique.

Dans la perspective que nous avons adoptée, celle de la syntagmatique de l'éthique, la différence entre le cas de l'homme qui ne se pique de rien et celui des actes manqués tient au traitement inégal des deux directions du lien entre l'opérateur et son lien: dans le premier cas, la responsabilité et l'imputation ( $2 \mathrm{a}$ et $2 \mathrm{~b}$ ) sont également affaiblies, alors que, dans le second cas, la responsabilité (2a) est affaiblie ou suspendue et l'imputation (2b) renforcée, en quelque sorte, par compensation. Cette analyse ouvre une nouvelle piste pour l'étude des freinages et des ruptures: les opérations éthiques, en effet, peuvent renforcer ou affaiblir soit l'ensemble d'un lien, dans les deux directions, soit chacune de ses directions séparément: on peut alors invoquer l'orientation converse ou inverse des opérations portant sur le lien axiologique.

Quelques articulations majeures de l'éthique praxique

Le principe selon lequel chaque modification d'un lien entraîne une modification d'un ou de plusieurs autres invite à considérer ces phénomènes de consistance comme un ensemble de tensions solidaires entre les liens: la consistance de la scène pratique, en effet, implique un jeu de compensations entre liens, l'ensemble des opérations fonctionnant, comme on l'a déjà indiqué, «à somme nulle». Ce principe nous permet d'envisager l'ethos comme étant celui de la scène pratique toute entière, et non seulement celui de l'opérateur. Toutefois, l'approche holistique, aussi justifiée soit-elle, ne suffit pas, et il faut pouvoir examiner plus précisément la distribution des valeurs éthiques sur chacune des instances de la pratique. Le principe général des tensions de la consistance pourra alors être décliné en tensions locales.

L'exploration systématique des articulations du champ de l'éthique est un vaste projet de recherches, que nous ne pouvons qu'ébaucher ici. La méthode consiste à adopter successivement le point de vue de l'un des trois actants, ou de l'acte lui-même, de manière à pouvoir qualifier les situations éthiques induites par les tensions dont chacun d'eux est le centre organisateur. Notons cependant que cette méthode ne permet pas pour l'instant de rendre compte de manière systématique des opérations inverses portant sur un même lien et que cet aspect des opérations éthiques devra être examiné dans un second temps de la recherche.

Choisissons ici, pour commencer, et dans les limites de cet article, le point de vue de l'opérateur: cette réduction provisoire permet d'entamer, d'abord déductivement, la construction du modèle et d'en vérifier ainsi au moins la vraisemblance et la cohérence. On sélectionne donc les trois liens centrés sur l'opérateur:

(i) les tensions entre le lien "opérateur/objectif» et le lien «opérateur/acte»,

(ii) celles entre le lien «opérateur/objectif» et le lien "opérateur/horizon stratégique»,

(iii) celles entre le lien "opérateur/acte» et le lien «opérateur/horizon stratégique».

Il résulte de cette proposition, grâce au déploiement de trois structures tensives, trois typologies complémentaires qui permettent une première ébauche d'articulation du champ de l'éthique praxique. 
1. Tensions éthiques entre "opérateur/objectif» et "opérateur/acte». L'évaluation de la «mobilisation pratique» de l'opérateur

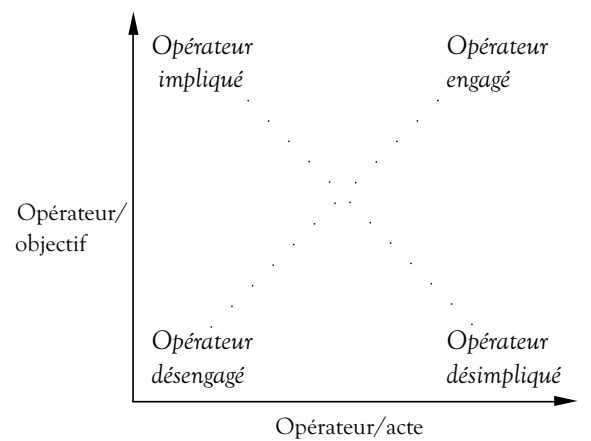

2. Tensions éthiques entre "opérateur/objectif» et "opérateur/horizon stratégique».

L'évaluation de la "projection" pratique de l'opérateur

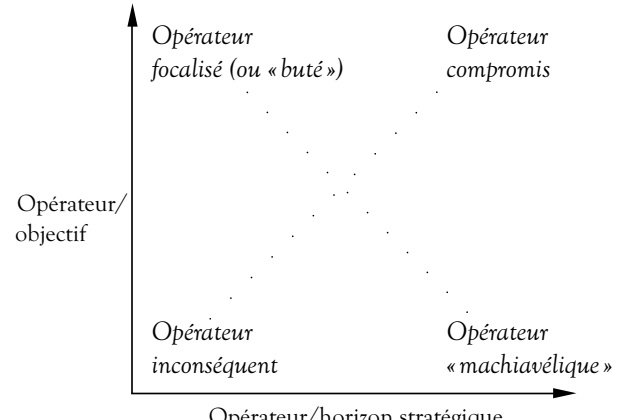

3. Tensions éthiques entre "opérateur/acte» et "opérateur/horizon stratégique».

L'évaluation de la "morale pratique» de l'opérateur

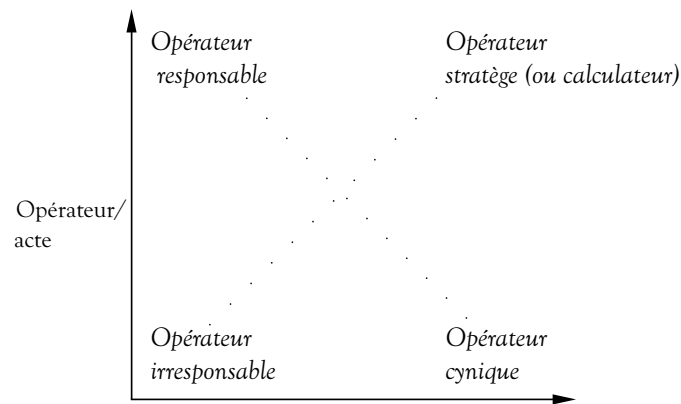

Opérateur/horizon stratégique

\section{CONCLUSION}

L'étymologie et le sens commun invitent à rapprocher, si ce n'est à confondre, l'ethos (møurs) et la pratique (usages, habitudes, normes, etc.). Une analyse sémiotique plus précise les distingue, pourtant, en limitant le domaine de pertinence à la forme prédicative de la scène praxique et, pour finir, circonscrit le champ de l'éthique aux opérations portant sur la consistance de cette scène.

Du point de vue des pratiques, qui semble le plus pertinent actuellement pour caractériser la dimension éthique, celle-ci est donc essentiellement une question de «bonne forme» syntagmatique, pour ne pas dire de «bonnes manières", que les usages concrets mettent à rude épreuve.

En effet, comme bien souvent dans les sciences humaines, la construction d'un modèle ne débouche sur une pratique d'analyse heuristique que si ce modèle prévoit les conditions de sa variation et de sa transgression. Le modèle de la scène praxique et de ses liens axiologiques ne déroge pas à cette règle: d'un côté, la «bonne forme» syntagmatique fournit à la fois les conditions de la scénarisation, et donc de la reconnaissance de la pratique en tant que «scène» signifiante, et un référentiel pour l'ensemble des opérations éthiques; mais, de l'autre côté, ces opérations, les freinages et les ruptures, ont pour vertu principale de pouvoir en tous sens modifier, déformer le modèle, compromettre la stabilité générale de la forme canonique et tester jusqu'à la rupture la consistance des liens qui la constituent. 


\section{N O TES}

1. Landowski (2004 et 2006). Une précision s'impose: Eric Landowski ne distingue pas la dimension esthétique et la dimension éthique, il les fusionne volontairement dans un même déploiement des esthésies interactives.

2. Voir l'entrée «Idéologie» dans Greimas et Courtés (1979: 179).

3. Toutes les mentions et propositions qui renvoient dans cette étude aux travaux de Perelman font référence à cet ouvrage. Nous ne préciserons pas les pages à chaque évocation de la théorie de Perelman, puisque chacune suppose la connaissance entière de l'ouvrage mentionné.

4. Les "procédures» sont une des formes syntagmatiques de la "praxis", à côté, notamment, des conduites, des protocoles et des rituels. Elles se caractérisent, spécifiquement, par la modalité dominante du «savoir-faire».

5. Nous préférerons désormais ce dernier terme à celui de "dissociation", utilisé par Perelman.

6. Ce sont ces éléments non textuels qu'on identifie traditionnellement, quand on adopte le niveau de pertinence du texte, comme des éléments "contextuels".

7. Cette remarque renvoie à la structure de la manipulation, dont le «faire» a pour objet un autre «faire»: $\mathrm{A}$ «fait faire » à B quelque chose, et ce "faire quelque chose" est le noyau prédicatif de l'"autre scène ". 8. Daniel Patte justifie le qualificatif "éthique" de ces combinaisons modales par le seul fait qu' «elles jouent un rôle important dans les discours éthiques»; cela ne suffit pas, évidemment, à fonder une dimension éthique en sémiotique.

9. Voir P. Ricœur (1990) et J.-M. Floch (1995: 34-41).

10. Voir à ce sujet E. Landowski (2004 et 2006).

11. Voir notamment R. Jakobson (1963: 213-220).

12. Cette remarque implique, indirectement, que la question de la modalisation (distance, subjectivation, etc.), abusivement limitée, dans les années 1970, à l'énonciation, devrait être traitée tout aussi bien dans une sémantique (et une sémiotique) de l'action (et des passions): de fait, cette restriction montre bien l'ostracisme dont l'éthique a été victime: en effet, en réduisant par exemple le "détachement " à une attitude énonciative, on peut le décrire en termes de "degré de subjectivité», dans la tradition ouverte par Benveniste; mais quand le détachement devient celui de n'importe quel opérateur, engagé dans n'importe quelle pratique, et surtout dans une pratique non verbale, il devient difficile de le limiter à un effet de subjectivité, et l'éthique réclame alors sa place.

13. Dans le cas de la pratique communicationnelle, évoquée plus haut, le lien phatique participe lui aussi spécifiquement de la scénarisation et de la consistante iconique de la scène de l'échange. Un dialogue sans lien phatique (et, par conséquent, sans "coopération " énonciative) est alors considéré comme inconsistant, non reconnaissable, voire paradoxal, comme en témoigne le sens commun dans l'expression «dialogue de sourds".

14. On pourrait faire la même analyse de la plupart des tropes: de même que les déplacements métonymiques remettent en cause non pas les relations structurelles propres à une scène ou à une situation donnée, mais la consistance des liens qui lui donnent forme, on pourrait dire tout autant que les «substitutions» métaphoriques ne provoquent pas de véritables "ruptures d'isotopie», mais se contentent d'éprouver l'élasticité du lien qui constitue l'isotopie.

\section{RÉFÉREN CES BIBLIO G RAPHIQ U ES}

BENVENISTE, É. [1966]: Problèmes de linguistique générale, tome 1, Paris, Gallimard.

FLOCH, J.-M. [1995]: Identités visuelles, Paris, PUF. FonTANILLE, J. [2003] : «Valeurs rhétoriques», Tópicos del Seminario, $\mathrm{n}^{\circ} 8$, Puebla, Mexique;

[2006]: «Pratiques sémiotiques: immanence et pertinence, efficience et optimisation ", Nouveaux Actes Sémiotiques, n 104-105-106, Limoges, PULIM, 13-73;

[ [2007]: «Textes, objets, situations et formes de vie. Les niveaux de pertinence du plan de l'expression dans une sémiotique des cultures", dans J. Alonzo, D. Bertrand, M. Costantini et S. Dambrine (dir.), Transversalité du Sens. Parcours sémiotiques, Saint-Denis, Presses universitaires de Vincennes, coll. «Essais et savoirs».

GenETTE, G. [1973]: Figures III, Paris, Seuil.

Greimas, A.J. [1970]: Du Sens. Essais sémiotiques, Paris, Seuil. Greimas, A.J. et J. CourTÉs [1979]: Sémiotique. Dictionnaire raisonné de la théorie du langage, Paris, Hachette.

JAKOBSON, R. [1963] : Essais de linguistique générale 1, Paris, Minuit. JOST, F. [(2001) 2003]: La Télévision du quotidien. Entre réalité et fiction, Bruxelles et Paris, De Boeck Université/INA, coll. «Médias

Recherches".

LANDOWSKI, E. [2004]: Passions sans nom, Paris, PUF;

[2006]: «Interactions risquées ", Nouveaux Actes Sémiotiques, no 101-102-103, Limoges, PULIM, 7-100.

PATTE, D. [1986] : "Modalités éthiques", dans A. J. Greimas et

J. Courtés, Dictionnaire raisonné de la théorie du langage. Sémiotique II, Paris, Hachette, 80-81.

PERELMAN, C. [1988]: Traité de l'argumentation. La nouvelle rhétorique, Bruxelles, Éd. de l'Université Libre de Bruxelles.

Ricceur, P. [1990]: Soi-même comme un autre, Paris, Seuil. 